\title{
Emergência do novo coronavírus (SARS-CoV-2) e o papel de uma vigilância nacional em saúde oportuna e efetiva
}

\author{
The novel coronavirus (SARS-CoV-2) emergency \\ and the role of timely and effective national \\ health surveillance
}

\section{Surgimiento del nuevo coronavirus (SARS-CoV-2) y el papel de una vigilancia nacional de la salud oportuna y eficaz}

\author{
Raquel Martins Lana 1 \\ Flávio Codeço Coelho 2 \\ Marcelo Ferreira da Costa Gomes 1 \\ Oswaldo Gonçalves Cruz 1 \\ Leonardo Soares Bastos 1,3 \\ Daniel Antunes Maciel Villela 1 \\ Cláudia Torres Codeço 1
}

doi: 10.1590/0102-311X00019620

Coronavírus são RNA vírus causadores de infecções respiratórias em uma variedade de animais, incluindo aves e mamíferos 1. Sete coronavírus são reconhecidos como patógenos em humanos. Os coronavírus sazonais estão em geral associados a síndromes gripais. Nos últimos 20 anos, dois deles foram responsáveis por epidemias mais virulentas de síndrome respiratória aguda grave (SRAG). A epidemia de SARS que emergiu em Hong Kong (China), em 2003, com letalidade de aproximadamente 10\% 2 e a síndrome respiratória do Oriente Médio (MERS) que emergiu na Arábia Saudita em 2012 com letalidade de cerca de 30\%. Ambos fazem parte da lista de doenças prioritárias para pesquisa e desenvolvimento no contexto de emergência 3.

O novo coronavírus, denominado SARS-CoV-2, causador da doença COVID-19, foi detectado em 31 de dezembro de 2019 em Wuhan, na China. Em 9 de janeiro de 2020, a Organização Mundial da Saúde (OMS) confirmou a circulação do novo coronavírus. No dia seguinte, a primeira sequência do SARS-CoV-2 foi publicada por pesquisadores chineses. Em 16 de janeiro, foi notificada a primeira importação em território japonês. No dia 21 de janeiro, os Estados Unidos reportaram seu primeiro caso importado. Em 30 de janeiro, a OMS declarou a epidemia uma emergência internacional (PHEIC) 4. Ao final do mês de janeiro, diversos países já haviam confirmado importações de caso, incluindo Estados Unidos, Canadá e Austrália. No Brasil, em 7 de fevereiro, havia 9 casos em investigação, mas sem registros de casos confirmados 5 .

A velocidade de propagação de uma doença pode ser avaliada pelo seu número básico de reprodução $\left(\mathrm{R}_{0}\right)$, definido como o número médio de casos secundários gerados por caso primário. As estimativas iniciais de $\mathrm{R}_{0}$ para o SARS-CoV-2 variam de 1,6 a 4,1 6,7,8. Para comparação, a epidemia de Influenza A H1N1 2009 apresentou $R_{0}$ entre 1,3 e 1,8 9, alcançando uma taxa de ataque de 643 casos por 100 mil no Estado do Paraná (de maior notificação), ficando entre 50 e 70/100 mil nos demais estados do Sudeste. Como o SARS-CoV-2 tem uma transmissibilidade maior, a introdução deste no Brasil, em condições semelhantes às do vírus Influenza, resultaria em uma taxa de ataque também maior. A predição do impacto em internação e mortalidade, porém, depende de informações sobre proporção de casos graves e letalidade, ainda desconhecidas. Os primeiros achados sugerem que a letalidade seja menor do que a do H1N1 e de outros coronavírus. Até 9 de fevereiro de 2020, dos 37.251 casos confirmados na China, 6.188 (16,6\%) foram classificados como graves e 812 resultaram em óbitos $(2,2 \%$ no geral e 13,2\% entre os casos graves) 10. Para termos uma comparação de magnitude, nos anos de 2018 e 2019, a letalidade observada entre casos de SRAG por Influenza notificados no Brasil foi da
1 Programa de Computação Científica, Fundação Oswaldo Cruz, Rio de Janeiro, Brasil.

2 Escola de Matemática Aplicada, Fundação Getúlio Vargas, Rio de Janeiro, Brasil.

${ }^{3}$ Department of Infectious Disease Epidemiology, London School of Hygiene and Tropical Medicine, London, U.K.

\section{Correspondência} R. M. Lana

Programa de Computação Científica, Fundação Oswaldo Cruz.

Av. Brasil 4365, Rio de Janeiro, RJ 21040-900, Brasil.

raquelmlana@gmail.com 
ordem de $20 \%{ }^{9}$. A letalidade do SARS-CoV-2, até o momento, tem sido majoritariamente associada a pacientes idosos ou à presença de comorbidades que afetam o sistema imunológico ${ }^{11}$. No entanto, a epidemia ainda está em um estágio inicial de evolução e registro de casos, com relativamente poucos estudos clínicos e com muitos casos ainda hospitalizados; portanto, esse quadro ainda é preliminar.

Nos últimos anos, a emergência e reemergência de doenças infecciosas, como gripe aviária (Influenza A H5N1) em 2003, a SRAG em 2002/2003, a Influenza A H1N1 em 2009, a Zika em 2015, suscitaram muitas questões sobre o papel da vigilância epidemiológica. Pandemias têm ocorrido com frequência maior, e a partir de 2018, a OMS reconheceu a necessidade de preparação antecipada à emergência de novos patógenos, incluindo, sob o nome de "doença X", as doenças ainda desconhecidas com potencial de emergência internacional na lista de prioridades para pesquisa e desenvolvimento no contexto de emergência 3,12. A emergência de novas doenças traz impactos muito além dos casos e mortes que geram. Elas criam também um contexto ideal que impõe aos sistemas nacionais de saúde pública a tarefa de validar seu sistema de vigilância e assistência em saúde quanto à oportunidade de detecção precoce e ao poder de resposta que vem em cascata.

Nos últimos 20 anos, o Brasil tem realizado avanços importantes na vigilância epidemiológica. Em 2003, o vírus Influenza A H5N1 foi a motivação para a elaboração do primeiro Plano de Contingência para Pandemia de Influenza 13. Esse Plano definiu as diretrizes para o fortalecimento da vigilância epidemiológica do país com a instituição de redes de laboratórios e de unidades sentinelas de síndromes respiratórias agudas graves, rede nacional de alerta e resposta às emergências em saúde, os CIEVS (Centro de Informações Estratégicas e Resposta de Vigilância em Saúde), além de investimentos na produção nacional de vacina contra influenza 14. Alguns anos depois, em 2009, a chegada do vírus Influenza A H1N1 encontrou uma rede mais estruturada que conseguiu responder com uma vigilância eficiente, pelo menos em algumas Unidades da Federação 15. O Estado de São Paulo naquela época destacou-se pela sua capacidade laboratorial, enquanto o Paraná, pela sensibilidade de sua rede. Essa experiência permitiu melhoria nos laboratórios para a tipagem dos subtipos virais e ampliação dos testes realizados, além da expansão da rede de vigilância de SRAG no país. Em 2015, o Brasil se posicionou no mapa da ciência mundial, sendo protagonista no avanço do conhecimento sobre o vírus Zika 16 .

Até a chegada do SARS-CoV-2, o protocolo de vigilância de SRAG no Brasil não incluía os coronavírus como parte do painel de exame laboratorial na rotina da vigilância, sendo explorado apenas em casos de óbitos e surtos por parte dos Laboratórios Nacionais de Influenza (NICs). A exceção é o Estado do Paraná, cujo Laboratório Central de Saúde Pública (LACEN) inclui no seu painel de RT-PCR os tipos sazonais. Em 2019, dos mais de 5 mil casos de SRAG notificados no estado, apenas 160 apresentaram resultado positivo para coronavírus (dados do SIVEP-gripe, Ministério da Saúde, acessado em 14/Jan/2020).

Mediante a situação posta pelo novo SARS-CoV-2, em 31 de janeiro de 2020, o Ministério da Saúde do Brasil instaurou o Grupo de Trabalho Interministerial de Emergência em Saúde Pública de Importância Nacional e Internacional para acompanhamento da situação e definição de protocolos de ação 17,18 , para a vigilância do SARS-CoV-2 no país. O protocolo estabeleceu a coleta de duas amostras para todos os pacientes atendidos na rede pública de saúde que atendam a definição de caso, que leva em conta não apenas o quadro sintomático característico, como também o histórico de viagem recente às regiões que apresentam transmissão direta e/ou histórico de contato com caso suspeito ou confirmado 5 . As amostras coletadas devem ser processadas pelos LACENs para triagem, testando para vírus respiratórios que fazem parte do painel da vigilância de SRAG, ficando a critério dos estados estabelecer também o teste local para COVID-19. Os casos negativos ou inconclusivos serão processados pelos NICs para teste específico para COVID-19, sendo realizado em paralelo análise de metagenômica. Além disso, a fim de minimizar o impacto do atraso de notificação e digitação, estabeleceu-se também canais prioritários de notificação, sem necessidade da notificação hierárquica (município/estado/federação), e plataforma de visualização rápida para a divulgação dos casos suspeitos 19, a Plataforma Integrada de Vigilância em Saúde (Plataforma IVIS - http://plataforma.saude. gov.br/novocoronavirus/). 


\section{Desafios postos para melhorar a efetividade da resposta à COVID-19}

A potencial chegada do novo vírus coloca à prova a estrutura de vigilância existente no país, principalmente num momento em que a redução de investimentos no Sistema Único de Saúde (SUS) e na pesquisa fragiliza a capacidade de detecção precoce e de resposta. O Brasil, que foi protagonista na epidemia de Zika, precisa acompanhar o avanço de conhecimentos gerados no exterior e preparar-se para as pesquisas e demandas específicas que surgirão no país, incluindo diagnóstico, assistência, prevenção e promoção da saúde. Portanto, quando o alerta para o novo coronavírus foi disparado, mais uma vez pairou a grande pergunta no território brasileiro: estamos preparados?

O esforço mundial de geração de informações sobre o novo coronavírus é impressionante. Em um mês de existência, o novo vírus já era citado em 37 publicações no PubMed, com análises descritivas dos primeiros casos, análises de sequências genômicas e aspectos clínicos. Esse movimento é produto de um sistema de vigilância internacional sensível, assim como de uma política de compartilhamento de dados e achados. Enquanto alguns grupos rapidamente se organizaram para monitorar casos em tempo real, outros se empenharam na aplicação de modelos matemáticos e estatísticos para monitorar o novo vírus e definir estratégias de ação 20,21,22.

Em contrapartida, o avanço do uso de mídias sociais como meio de informação trouxe consigo o desafio de monitorar e responder rapidamente a conteúdos falsos disseminados nestes canais, e de forma que possam igualmente circular nos mesmos. Por exemplo, em paralelo às notícias oficiais e matérias informativas em veículos tradicionais, áudios falsos com recomendações equivocadas circularam em mídias sociais se passando por comunicado de entidades de respaldo público como a Sociedade Brasileira de Infectologia (SBI) 23 . Também houve a tentativa de resgatar o mito de que certos chás têm as mesmas propriedades antivirais do fosfato de oseltamivir (princípio ativo do antiviral usado para o tratamento de SRAG por vírus Influenza), sugerindo o consumo destes para casos de influenza e coronavírus 24. Ambos ensejaram notas de esclarecimento por parte da SBI e do Ministério da Saúde, porém com alcance desconhecido. Dentro desse contexto, o crescente movimento de descrédito dos canais tradicionais de comunicação, que fomenta a adesão a fontes alternativas, tornase também um risco à saúde pública que deve ser enfrentado. A comunicação de especialistas não pode ficar restrita ao ambiente acadêmico e profissionais da área.

No âmbito da vigilância laboratorial é de fundamental importância que os LACENs contem com estoque de insumos que os mantenham constantemente capazes de processar as amostras recebidas e liberar os resultados em tempo oportuno. O desabastecimento, seja de kits para a detecção de agentes (primers, sondas, controle etc.), seja de pessoal capacitado, atrasa a liberação de resultados produzidos localmente ou exige o envio para os NICs, gerando não apenas atraso na notificação, como sobrecarga nos laboratórios de referência. Sem esses insumos, o investimento em instalação de máquinas de processamento de qualidade, embora fundamental, torna-se inócuo. A liberação oportuna de resultados laboratoriais é de suma importância para a vigilância de casos inusitados, como surto por novos agentes infecciosos e surtos antecipados de doenças endêmicas sazonais como influenza e arboviroses.

No âmbito do processamento de dados, o compartilhamento e análise oportuna de dados epidemiológicos no Brasil ainda enfrentam desafios apesar dos avanços nas políticas de transparência como o e-SIC (Sistema Eletrônico do Serviço de Informações ao Cidadão) e o investimento nos últimos anos em sistemas de acompanhamento em tempo real de situação de alerta, como o InfoGripe 9 . Dentre os principais desafios, citamos a infraestrutura heterogênea que o sistema de vigilância em saúde tem, uma vez que a qualidade e a oportunidade da informação dependem primordialmente da redução do "atrito" à entrada dos dados no sistema. Em muitas localidades ainda se preenchem fichas em papel que precisam ser acumuladas e digitadas. A falta de validação dos dados no momento do preenchimento dos formulários eletrônicos leva à entrada de dados incorretos que poderiam ser automaticamente corrigidos no momento da digitação.

Outro ponto crítico é a falta de integração entre diferentes sistemas de informação existentes, o que torna inviável a integração de informações de diferentes fontes. Por exemplo, o Sistema Gerenciador de Ambiente Laboratorial (GAL) não tem o número da notificação feita no Sistema de Informação de Agravos de Notificação (SINAN), impossibilitando o acompanhamento dos resultados dos exames em tempo real, atrasando a detecção precoce do caso, além de contribuir para falhas no preenchimento das notificações, que muitas vezes ficam sem o fechamento do caso. Na perspectiva do analista da 
informação, dificuldades no tocante ao acesso aos dados levam a frequentes blackouts de dados além de grande esforço na reconstrução da informação, carregando incertezas para as análises.

A frequente emergência de novos agravos exige uma reestruturação na forma como doenças são notificadas no país. É preciso investir em um novo SINAN, baseado em tecnologias mais modernas tanto para facilitar a notificação como para permitir a disseminação e análise de dados de uma maneira mais célere, aderente aos princípios da epidemiologia de precisão 25. É fundamental que o Ministério da Saúde desenvolva uma infraestrutura integrada de dados à altura da velocidade de espalhamento das doenças nesta era de alta mobilidade global. Há de se considerar um sistema flexível o suficiente para permitir a entrada de novos agravos, mas sem perder a estrutura existente. Quanto à comunicação desse tipo de informação e disponibilização dos dados, o acesso por APIs ao sistema de notificação é fundamental, uma vez que possibilita a construção de dashboards e relatórios automatizados para o acompanhamento temporal e espacial dos casos notificados e confirmados com o mínimo de atraso. O exemplo positivo dos canais rápidos de notificação e visualização implementados para o surto atual, reconhecidamente fundamental para as ações oportunas, deveria ser incorporado como o padrão da vigilância epidemiológica nacional.

\section{Colaboradores}

R. M. Lana, F. C. Coelho e C. T. Codeço contribuíram com a concepção, redação e revisão do artigo. M. F. C. Gomes, O. G. Cruz, L. S. Bastos e D. A. M. Villela contribuíram com a redação e revisão do artigo.

\section{Informações adicionais}

ORCID: Raquel Martins Lana (0000-0002-75731364); Flávio Codeço Coelho (0000-0003-38684391); Marcelo Ferreira da Costa Gomes (00000003-4693-5402); Oswaldo Gonçalves Cruz (00000002-3289-3195); Leonardo Soares Bastos (00000002-1406-0122); Daniel Antunes Maciel Villela (0000-0001-8371-2959); Cláudia Torres Codeço (0000-0003-1174-178X).

\section{Agradecimentos}

Rede Nacional de Vigilância de Influenza (LACENs, vigilâncias estaduais e municipais, e GT-Influenza, Departamento de Vigilância das Doenças Transmissíveis da Secretaria de Vigilância em Saúde do Ministério da Saúde e NICs) pela parceria.

\section{Referências}

1. Fehr AR, Perlman S. Coronaviruses: an overview of their replication and pathogenesis. Methods Mol Biol 2015; 1282:1-23.

2. World Health Organization. Severe acute respiratory syndrome (SARS). https://www.who. int/csr/sars/en/ (acessado em 03/Fev/2020).

3. World Health Organization. Prioritizing diseases for research and development in emergency contexts. https://www.who.int/activi ties/prioritizing-diseases-for-research-anddevelopment-in-emergency-contexts (acessado em 29/Jan/2020).

4. World Health Organization. IHR procedures concerning public health emergencies of international concern (PHEIC). http://www.who. int/ihr/procedures/pheic/en/ (acessado em 29/Jan/2020).

5. Secretaria de Vigilância em Saúde, Ministério da Saúde. Infecção humana pelo novo coronavírus (2019-nCoV). Boletim Epidemiológico 2020; (02). https://portalarquivos2.saude.gov. br/images/pdf/2020/fevereiro/07/BE-COECoronavirus-n020702.pdf.

6. Read JM, Bridgen JRE, Cummings DAT, Ho A, Jewell CP. Novel coronavirus 2019-nCoV: early estimation of epidemiological parameters and epidemic predictions. medRxiv 2020; 28 jan. https://www.medrxiv.org/content/10.110 1/2020.01.23.20018549v2.

7. Liu T, Hu J, Kang M, Lin L, Zhong H, Xiao J, et al. Transmission dynamics of 2019 novel coronavirus (2019-nCoV). BioRxiv 2020; 26 jan. https://www.biorxiv.org/conten t/10.1101/2020.01.25.919787v1.

8. Cao Z, Zhang Q, Lu X, Pfeiffer D, Jia Z, Song $\mathrm{H}$, et al. Estimating the effective reproduction number of the 2019-nCoV in China. medRxiv 2020; 29 jan. https://www.medrxiv.org/con tent/10.1101/2020.01.27.20018952v1. 
9. InfoGripe. Situação da gripe. http://info.gripe. fiocruz.br/ (acessado em 03/Fev/2020).

10. World Health Organization. Novel coronavirus (2019-nCoV): situation reports - 20. https://www.who.int/docs/default-source/coro naviruse/situation-reports/20200209-sitrep20-ncov.pdf?sfvrsn=6f80d1b9_4 (acessado em 10/Fev/2020)

11. World Health Organization. Q\&A on coronaviruses. https://www.who.int/news-room/ q-a-detail/q-a-coronaviruses (acessado em 10/ $\mathrm{Fev} / 2020$ ).

12. Cousins S. WHO hedges its bets: the next global pandemic could be disease X. BMJ 2018; 361:k2015.

13. Secretaria de Vigilância em Saúde, Ministério da Saúde. Plano brasileiro de preparação para enfrentamento de uma pandemia de influenza. http://bvsms.saude.gov.br/bvs/publicacoes/ plano_brasileiro_pandemia_influenza_IV.pdf (acessado em 31/Jan/2020).

14. Costa LMC, Merchan-Hamann E. Influenza pandemics and the structure of Brazilian health care system: brief history and characterization of the scenarios. Rev Pan-Amazônica Saúde 2016; 7:11-25.

15. Codeço CT, Cordeiro JS, Lima AWS, Colpo RA, Cruz OG, Coelho FC, et al. The epidemic wave of influenza A (H1N1) in Brazil, 2009. Cad Saúde Pública 2012; 28:1325-36.

16. Brasil P, Pereira Jr. JP, Moreira ME, Nogueira RMR, Damasceno L, Wakimoto M, et al. Zika virus infection in pregnant women in Rio de Janeiro. N Engl J Med 2016; 375:2321-34.

17. Ministério da Saúde. Coronavírus e novo coronavírus: o que é, causas, sintomas, tratamento e prevenção. https://www.saude.gov.br/ saude-de-a-z/coronavirus (acessado em 03/ Fev/2020).
18. Ministério da Saúde. Grupo Interministerial vai atuar no enfrentamento ao novo corona vírus. http://saude.gov.br/noticias/agenciasaude/46266-grupo-interministerial-vaiatuar-no-enfrentamento-ao-novo-coronavi rus (acessado em 04/Fev/2020).

19. Plataforma Integrada de Vigilância em Saúde, Ministério da Saúde. Notificação de casos pelo novo coronavírus (COVID-2019). http://plata forma.saude.gov.br/novocoronavirus/ (acessado em 04/Fev/2020).

20. Johns Hopkins University. Coronavirus COVID-19 global cases by Johns Hopkins CSSE. https://gisanddata.maps.arcgis.com/apps/ opsdashboard/index.html\#/bda7594740f d40299423467b48e9ecf6 (acessado em 04/ Fev/2020).

21. MOBS Lab. Preliminary analysis of the 2019 nCOV outbreak in Wuhan city. https://www. mobs-lab.org/2019ncov.html (acessado em 04/Fev/2020).

22. Bedford T, Neher R, Hadfield J, Hodcroft E, Ilcisin M, Müller N. Genomic analysis of nCoV spread. Situation report 2020-01-23. https://nextstrain.org/narratives/ncov/si t-rep/2020-01-23 (acessado em 04/Fev/2020).

23. Sociedade Brasileira de Infectologia. Nota de repúdio (fake news). https://ammg.org.br/wpcontent/uploads/NotaRepu\%CC\%81dio_So ciedade-Brasileira-de-Infectologia.pdf (acessado em 10/Fev/2020).

24. Ministério da Saúde. Chá de erva doce e o tratamento do novo coronavírus - É FAKE NEWS! https://www.saude.gov.br/fakene ws/46239-cha-e-o-tratamento-do-novocoronavirus-e-fake-news (acessado em 10/ $\mathrm{Fev} / 2020$ ).

25. Coelho FC, Codeço CT. Precision epidemiology of arboviral diseases. J Public Health Emerg 2019; 3:1
Recebido em 04/Fev/2020

Versão final reapresentada em 12/Fev/2020

Aprovado em 13/Fev/2020 